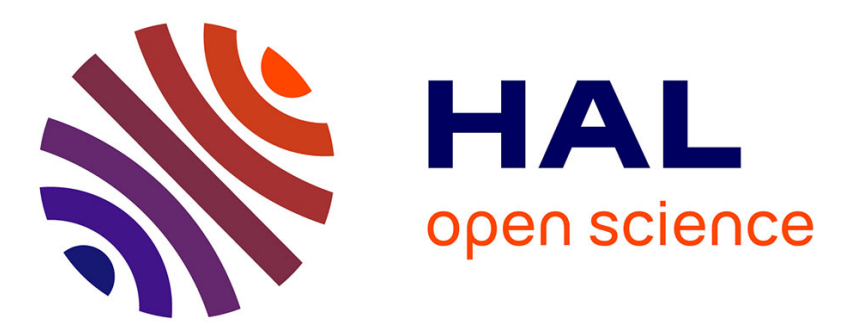

\title{
Measuring photon numbers in a cavity by atomic interferometry: optimizing the convergence procedure
}

\author{
S. Haroche, M. Brune, J. Raimond
}

\section{To cite this version:}

S. Haroche, M. Brune, J. Raimond. Measuring photon numbers in a cavity by atomic interferometry: optimizing the convergence procedure. Journal de Physique II, 1992, 2 (4), pp.659-670. 10.1051/jp2:1992157 . jpa-00247663

\section{HAL Id: jpa-00247663 https://hal.science/jpa-00247663}

Submitted on 1 Jan 1992

HAL is a multi-disciplinary open access archive for the deposit and dissemination of scientific research documents, whether they are published or not. The documents may come from teaching and research institutions in France or abroad, or from public or private research centers.
L'archive ouverte pluridisciplinaire HAL, est destinée au dépôt et à la diffusion de documents scientifiques de niveau recherche, publiés ou non, émanant des établissements d'enseignement et de recherche français ou étrangers, des laboratoires publics ou privés. 
Classification

Physics Abstracts

$42.50-03.65$

\title{
Measuring photon numbers in a cavity by atomic interferometry: optimizing the convergence procedure
}

\author{
S. Haroche, M. Brune and J.M. Raimond
}

Laboratoire de Spectroscopie Hertzienne de l'Ecole Normale Supérieure $\left(^{\star}\right), 24$ rue Lhomond, 75231 Paris Cedex 05 France

(Received 16 January 1992, accepted 23 January 1992)

Résumé . - Nous décrivons la procédure optimale pour mesurer par interférométrie atomique le nombre de photons contenus dans une cavité en utilisant une méthode quantique non-destructive. Il s'agit de celle décrite récemment par Brune et al. [1,2], qui consiste à détecter l'intensité du champ en enregistrant des interférences atomiques de type Ramsey. Une succession d'atomes à deux niveaux traverse la cavité qui se comporte comme une lame à retard biréfringente pour la fonction d'onde atomique. L'information est acquise sous la forme d'une suite de bits (atomes détectés dans un niveau ou dans l'autre). Nous montrons qu'une stratégie optimale permet d'obtenir le nombre de photons dans la cavité avec un nombre d' atomes détectés de l'ordre du logarithme à base 2 de la dispersion du nombre de photons dans le champ. La procédure décrite est en cours de réalisation expérimentale dans notre laboratoire.

\begin{abstract}
We describe the optimum detection procedure to follow in order to measure by atomic interferometry the number of photons present in a cavity by using a Quantum nondemolition scheme. The method we discuss is the one recently introduced by Brune et al. [1, 2], in which the field intensity is detected by recording atomic interferences in a Ramsey type set up. A succession of two-level atoms interacts with the cavity field which is the analog of a birefringent retarding plate for the atomic wave function. The information is acquired in the form of binary digits (atoms detected in one level or the other). We discuss an optimal strategy which yields the value of the photon number with a number of atoms of the order of the base 2 logarithm of the field photon number dispersion. The procedure described here is the one we intend to use in an experiment in preparation.
\end{abstract}

(*) Associé au Centre National de la Recherche Scientifique et à l’Université Pierre et Marie Curie. 


\section{Avant Propos / Foreword.}

Pierre Jacquinot est un des maîtres de l' Optique Française. Dans la tradition de Fabry et Cotton, il a, avec ses collaborateurs, inventé et développé un grand nombre de méthodes interférométriques nouvelles. Une grande part de son œuvre est spectroscopique et la Physique Atomique lui doit beaucoup. A la fin de sa carrière, il a été l'un des premiers à comprendre l'importance des lasers comme instruments d'investigation en physique et son laboratoire a grandement contribué à la mise au point de techniques puissantes de manipulation d'atomes et de spectroscopie non-linéaire et cohérente. Le nom de son collègue et ami Sylvain Liberman doit être associé à ce rappel de son œuvre. Pierre Jacquinot est également ce que les anglosaxons appellent un "statesman" de la science, un scientifique qui s'est toujours intéressé et dévoué au développement de la science française et internationale. Ses tâches lourdes et contraignantes ne l'ont d'autre part jamais éloigné de la recherche, ce qui n'est pas si fréquent. Enfin, il a toujours montré de l'intérêt et de la bienveillance pour le travail et la carrière de ses collègues plus jeunes. C'est donc à la fois un honneur et un plaisir d'avoir été sollicité pour écrire un article dans ce numéro du Journal de Physique qui lui est dédié.

Il est de bon ton, dans un tel exercice, d'essayer de relier le sujet de sa contribution aux centres d'intérêt du récipiendaire de l'hommage. Cela n'est pas très difficile dans le cas présent. Nous dirons que dans les expériences d'intérferométrie optique, dans lesquelles Pierre Jacquinot et son équipe se sont illustrés, on utilise de façon générale des interférences de la lumière pour acquérir de l'information sur un système matériel. Le développement récent de méthodes sophistiquées de manipulation des atomes et du champ rendent à présent possible des expériences pour ainsi dire duales des précédentes: des interférences affectant la fonction d'onde d'un système atomique peuvent maintenant ètre utilisées pour acquérir de l'information sur un champ quantique, le mesurer et le "manipuler" comme un véritable "objet". Un aspect important de toute expérience d'interférométrie est le problème de la visibilité des "franges": combien faut-il de photons pour acquérir une précision spectroscopique donnée ou encore, quelle intensité la source doit-elle avoir pour qu'un hologramme soit lisible? Un problème analogue se pose dans le cas de l'interférométrie atomique utilisée pour manipuler un champ quantique: la détection d'un nombre minimal d'atomes est nécessaire pour rendre les interférences de matière visibles et mesurer le champ. La situation que nous décrivons dans cet article est cependant moins banale qu'une simple analyse de rapport signal/bruit. L'objet mesuré est en effet intrinsèquement quantique et l'acquisition d'information sur le champ est un processus où le caractère probabiliste de la Mécanique Quantique joue un rôle essentiel. Néanmoins, l'analyse peut être menée "comme si" on avait affaire à un phénomène probabiliste classique...Nous espérons que cet aspect quelque peu inhabituel du problème du signal dans une expérience d'interférence atomique retiendra l'intérêt de Pierre Jacquinot.

\section{Introduction.}

We have recently proposed to measure by atomic interferometry the number $n$ of photons stored in a high $\mathrm{Q}$ cavity by using a quantum nondemolition process [1-3]. The method is based on a very simple idea: if an atom can follow two interfering "paths" along which the cavity field produces different shifts on the atomic energy levels, the detected atomic inteference pattern is phase-shifted by an amount proportional to the number of photons in the cavity. If this number is initially well defined, the observation of the atomic interference constitutes a simple determination of the field energy. More interestingly, if this number is not well defined, the atomic interferences, which are "revealed" after a large enough number of atoms have crossed 
the cavity, "fix" the photon number and the experiment constitutes a very simple illustration of the "collapse" of the field wave function into an eigenstate of the field energy.

An interesting aspect of this scheme is that the field coherences do not play any role in the photon number measurement process. As long as one is only interested in determining the field energy, all happens "as if" the cavity did contain initially a well defined number of photons "waiting" to be measured. This means that the measurement can be discussed formally as a classical "information gathering" process, which can be described by simple probability arguments. In this article, we adopt this point of view and analyze the photon number measurement process in classical terms. We describe a very simple scheme in which each atom interacting with the field can be considered as a two-level system whose measurement, after the interaction, provides a double valued "bit of information" (atom found in either one of the two levels). We will recall how this information can be used to determine the photon number in the cavity. We will describe various measurement strategies and determine the most economical way of performing a measurement, by using the smallest possible sample of atoms. The situation we analyse here corresponds to an experiment in preparation in our laboratory. We hope to show in this article that it constitutes a paradigm of a measurement process, providing a text book illustration of fundamental concepts of Quantum Mechanics. This paper consists in two main parts. We first remind the reader of the principle of our method and recall the simple formulae necessary to perform calculations. We then turn to the description of situations in which small photon numbers are measured and we describe an ideal procedure allowing to determine the photon number in the minimal number of steps compatible with information theory. We also compare this ideal procedure to other measurement strategies discussed elsewhere.

\section{A reminder of the method.}

The simplest way to explain our method is to use an analogy. We have sketched in figures 1a and $b$ two well known interference schemes, which can be used for optical as well as for matter wave. In order to emphasize the similarities, we use the same figures for optical and matter waves and label them at the top in the "optics language" and at the bottom in the "matter wave one". Figure 1a represents a Young double slit apparatus: in the optical version, a light wave impinges on a screen pierced with two small holes $S_{1}$ and $S_{2}$. The light scattered by these holes can follow two pathes to reach the detection plane and the probability of receiving a photon is a periodic function of position, characteristic of the interference between the two pathes. If, as shown on the figure, a retarding plate is introduced on one of the two channels, the corresponding optical amplitude is phase shifted, resulting in a spatial translation of the fringe pattern. Figure $1 \mathrm{~b}$ represents a different scheme, in which the interference affects not the external degrees of freedom of the field, but the photon or the atom polarization space: in the optical version, a light beam (wavelength $\lambda$ ) is linearly polarized along $\mathrm{O} x$ by the polariser $P_{1}$ and is sent through a transparent birefringent retarding plate whose fast and slow optical axes are $\mathrm{O} x$ and $\mathrm{O} y$. The plate is sandwiched between two optically active $45^{\circ}$ polarization rotators $R_{1}$ and $R_{2}$. The emerging light is finally transmitted through a linear polarization analyzer $\mathrm{P}_{2}$ parallel either to $\mathrm{O} x$ or $\mathrm{O} y$ and its intensity exhibits periodic variations versus the plate thickness. The retarding plate does indeed rotate the field polarization by $90^{\circ}$ when its thickness is an odd multiple of $\lambda / 2$ and by $180^{\circ}$ when it is a multiple of $\lambda$. As a result, the total polarization rotation angle is zero in the first case (maximum transmission through an $\mathrm{O} x$ linear analyser) and $90^{\circ}$ in the latter case (no transmission through the $\mathrm{O} x$ linear analyser, maximum transmission through the $\mathrm{O} y$ one). The periodic variations of the transmission, which are in phase-opposition through the $\mathrm{O} x$ and $\mathrm{O} y$ analysers, can again be understood as 
an interference effect. One merely has to remark that the photons enter the retarding plate in a linear superposition of its principal polarization states, which undergo different dephasings as they propagate through it. The probability for a photon to emerge from $R_{2}$ with the initial polarization appears as the squared sum of two amplitudes phase shifted with respect to each other by an amount proportional to the optical path length through the plate.
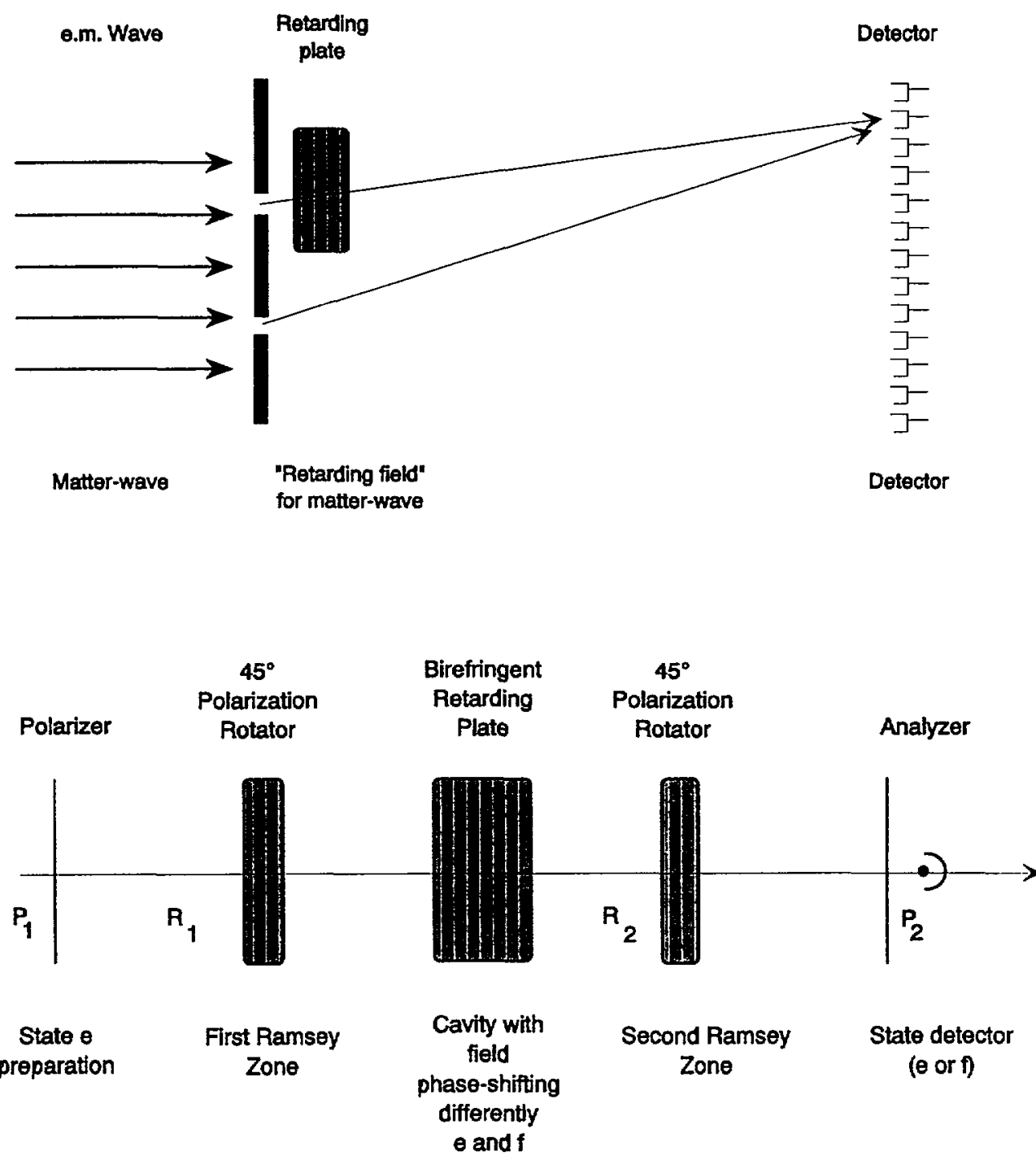

Fig. 1. - a) Young double slit experiment with a retarding plate in one of the arms (top: "optical language"; bottom: "matter-wave" language). b) Polarization interferometer (top: "optical case" representing the measurement of the thickness of a birefringent plate between polarization rotators; bottom: atomic version representing the measurement of a cavity field intensity by the Ramsey method). 
Let us now exchange the roles of matter and radiation and assume that atoms are sent through a region of space containing a "dispersive" field which is non resonant with any atomic transition, but phase-shifts the wave function of the atom by an amount proportional to the field intensity (i.e. to its photon number) and which also depends upon the quantum state in which the atom is prepared. With this "duality" in mind, figure 1a represents now a de Broglie wave Young double slit experiment (4), with a cavity containing the atomic-wave shifting field in one of its arms. Using the optical analogy, we expect in this case that the fringe pattern on the screen will be translated by an amount proportional to the photon number in the cavity, providing a conceptually simple way of measuring this number. The dual version of figure $1 \mathrm{~b}$, on the other hand, is a simple sketch of a Ramsey type experiment (5). The atoms are assumed to have two levels e and $f$ (transition frequency between them: $\omega_{\text {ef }}$ ). The atoms are prepared in level $\mathrm{e}$ in the "preparation zone" $\mathrm{P}_{1}$ (e.g. by laser excitation). The "atomic polarization rotators" $R_{1}$ and $R_{2}$ are two zones in which an auxiliary microwave field with angular frequency $\omega_{r}$ close to $\omega_{\text {ef }}$ is applied for a short time so as to transform e and $f$ into a linear superposition with equal weights of these states. The cavity between $R_{1}$ and $R_{2}$ contains the field to be measured, which phase shifts by different amounts levels e and $f$. After $R_{2}$, a selective detector $P_{2}$ measures the atoms in e or $\mathbf{f}$. All the ingredients of the optical birefringence interference experiment are gathered: we again predict that the probability of detecting the atom in level e or $f$ after zone $R_{2}$ exhibits oscillations versus the photon number in the cavity and we can use the detection of the atoms as a way to measure this number.

The Young and the Ramsey type experiments for measuring a photon number have been described in details in references (3) and (2) respectively. They are very closely related and lead to very similar calculations. In both cases the photon number is pinned down by detecting a large enough sample of atoms. In the Young experiment, this information takes the form of a sequence $\left[x_{1}, x_{2}, \ldots x_{k}\right]$ of atomic postions and in the Ramsey one it is a set of $\mathrm{e}$ and $\mathrm{f}$ detection outcomes, which can be conveniently replaced by a sequence of binary bits. In the reminder of this article, we will restrict our analysis to this latter case, for at least two good reasons. First, the Ramsey design is more "realistic" than the Young one and it is the one we are presently trying to demonstrate experimentally. Second, the Ramsey procedure is conceptually simpler to analyse since each atom provides the simplest kind of information one can think of (only two values instead of a continuum of values in the Young one).

Let us now summarize the quantitative results-borrowed from reference (2)- which will be useful for the analysis of a Ramsey experiment measuring $n$. The important parameter of this scheme is the differential phase-shift produced on levels e and $f$ as the atom crosses a cavity containing a single photon. The simplest way to produce such a shift is to tune the cavity close to a frequency corresponding to a transition $\mathrm{e} \rightarrow i$ linking e to a third level $i$, different from $\mathrm{f}$. The cavity field then induces a light shift on level e and not on $\mathrm{f}$. The differential phase shift between levels e and $f$ can be expressed as:

$$
\varepsilon=\left(\Omega^{2} / \delta\right) L / v
$$

In this expression, $\Omega$ is the vacuum Rabi frequency associated to the $e \rightarrow i$ transition and $\delta$ is the detuning between the cavity field and this transition, which must be large enough to avoid real photon absorption during the time the atom crosses the cavity. $L$ is the cavity length and $v$ the atom velocity. We have shown in (1) that circular Rydberg atoms and superconducting microwave cavities are systems in which $\varepsilon$ values of $1 \mathrm{rad}$ or more can easily be achieved.

After an atom initially in e has crossed $R_{1}$, the cavity containing $n$ photons and $R_{2}$, its quantum state simply writes:

$$
\left|\Psi_{\text {atom }}\right\rangle=\sum_{a} b_{a}(n, v, \varphi, \varepsilon)|a\rangle
$$


where $a$ stands for e or $\mathrm{f}$ and the $b_{a}$ 's are amplitudes, depending upon $n$, the atom velocity $v$, the phase shift per photon $\varepsilon$ and the detuning of the auxiliary Ramsey field, characterized by the phase:

$$
\varphi=\left(\omega_{\mathrm{r}}-\omega_{\mathrm{ef}}\right) L / v
$$

We will need in the following only the expression of the squared modulus of these amplitudes which are:

$$
\left|b_{\mathrm{f}}(n, v, \varphi, \varepsilon)\right|^{2}=1-\left|b_{\mathrm{e}}(n, v \varphi, \varepsilon)\right|^{2}=\sin ^{2}\left(\pi v_{0} / 2 v\right) \cos ^{2}\left(\frac{\varphi-n \varepsilon}{2}\right)
$$

where $v_{0}$ is the velocity for which each Ramsey zone produces an exact $\pi / 2$ rotation of the atomic state in the e $-\mathrm{f}$ space (equivalent of an exact $45^{\circ}$ polarization rotator in the optical analogy). Obviously $\left|b_{a}(n, v, \varphi, \varepsilon)\right|^{2}$ is the probability of detecting the atom in level $a$ when the cavity field contains $n$ photons. The cosine function in equation (4) is typical of an interference effect. The probabilities of finding the atom in e or $f$ exhibits "Ramsey fringes" as a function of $\varphi$, whose phase depends upon the number of photons present in the cavity. For a given $\varphi$ value, these probabilities also appear as oscillating functions of $n$.

Let us stress that the transitions between $e$ and $f$ are induced by the auxiliary Ramsey field, and not by the cavity field to be measured, which is non resonant. The fact that the transition probabilities depend upon $n$ is due to a dispersive effect which does not affect the photon number, hence the nondemolition character of the method.

Let us assume now that the field is initially in a quantum superposition:

$$
|\alpha\rangle=\sum_{n} C_{n}|n\rangle
$$

for which the number of photons exhibits fluctuations. The atom+field quantum state after the interaction of an atom with this field is simply obtained by using the linearity of Quantum Mechanics:

$$
\left|\Psi_{\text {atom }+ \text { field }}\right\rangle=\sum_{n} C_{n} \sum_{a} b_{a}(n, v, \varphi, \varepsilon)|n ; a\rangle
$$

After detection of the atom in level a (e or $f$ ) the system "collapses" into the state $\sum_{n} C_{n} b_{a}(n, v, \varphi, \varepsilon)|n ; a\rangle$, which means that the field amplitude $C_{n}$ has undergone the following simple transformation:

$$
C_{n} \rightarrow C_{n} b_{a}(n, v, \varphi, \varepsilon) /\left[\sum_{n^{\prime}}\left|C_{n^{\prime}}\right|^{2}\left|b_{a}\left(n^{\prime}, v, \varphi, \varepsilon\right)\right|^{2}\right]^{1 / 2}
$$

The atomic detection thus results in the multiplication of the photon probability $p(n)=\left|C_{n}\right|^{2}$ by the "fringe function" which characterizes the Ramsey interference. If $\varepsilon$ is well chosen, some $n$ values are efficiently decimated by the multiplication process. Detecting other atoms then results in multiplying again the resulting probability distribution by "fringe functions" with dark fringes located elsewhere and numerical simulations show that eventually $p(n)$ converges into a delta function of $n$ describing a Fock state.

An important point to stress at this stage is that the coherence between $n$ states is irrelevant in the photon number detection process. The evolution of $p(n)$ in a measuring sequence would be the same if we assume that the field, instead of being initially described by equation (5), is in a statistical mixture of Fock states described by the density operator:

$$
\rho=\sum_{n}\left|C_{n}\right|^{2}|n\rangle\langle n|
$$


Such a classical mixture can be seen as describing a field containing in each realization of the experiment a well defined- although not well known- number of photons. The interaction with the atoms crossing the cavity thus appears as a classical information gathering process which allows us to determine the initially unperfectly well known field intensity. Each measuring sequence determines the $n$ value of that particular realization and the histogram constructed with many such measuring sequences reproduces the initial $p(n)$ distribution.

The efficiency of the convergence, defined by the minimal number of atoms required to obtain it, is very important to study, both for conceptual and practical reasons. Fundamentally, it is interesting to design the most ideal procedure, which allows us to reduce the field with the smallest possible atomic sample. This is a simple question in terms of information theory: can we avoid redundant information and obtain $n$ with no more digits as required to express this integer in the binary basis? Practically, it is also very important to be able to make a measurement in the shortest possible time, since we must avoid field relaxation during the process. In the simple theoretical model outlined above, the field is supposed to evolve freely in an ideal lossless cavity. In a real experiment, a Fock state would decay in a characteristic time $T_{\text {cav }} / n$ where $T_{\text {cav }}$ is the cavity energy damping time and we will hardly have more than a fraction of a second to prepare and detect each Fock state. We have briefly discussed this question in reference [2] and shown by numerical simulations that the convergence is more efficient when we vary from atom to atom the phase-dephasing. A simple way to achieve this is to use atoms with random velocities, assuming of course that this velocity is measured for each detected atom (we need the value of $v$ to determine the "fringe function" at each step). The simulations show that with random velocities and a $\varepsilon$ value such that $\varepsilon \Delta n \simeq 1$ ( $\Delta n$ is the initial fluctuation of $n$ ), the convergence to a Fock state requires a number of atoms increasing logarithmically with $\Delta n$. The convergence is generally still possible when the atomic velocity (and all the other parameters in Eq.(4)) are kept fixed throughout the measurement, but it requires many more atoms. The reason for this is quite clear: when the parameters of the experiment are varied from atom to atom, the process efficiently decimates different photon numbers since the "dark fringes" of the Ramsey pattern are at each step translated. On the other hand, identical atoms, all submitted to the same dephasing, provide a lot of redundant information. Obviously, the use of atoms with random velocities is not the optimal situation we can think of. The velocities of two atoms may accidentally coincide, or nearly coincide, so that these atoms will again provide somewhat redundant information. Furthermore, values of $v$ different from $v_{0}$ result in a probability fringe contrast (difference between the maximum and the minimum probability as a function of $n$ ) smaller than unity, a situation which is less likely to allow for an optimal convergence of the process. An alternative way to obtain a variable $\varepsilon$ without having to change $v$ is to tune the atomic transition frequency (for example by applying an electric field Stark shifting the Rydberg levels in the cavity). This changes the value of $\delta$ in equation (1) and thus modifies $\varepsilon$. The velocity can then be kept fixed to the value $v_{0}$ corresponding to exact $\pi / 2$ pulses in each Ramsey zone, a situation which is easier to describe. Finally, it is also possible to tune $\varphi$ between-atoms by changing $\omega_{\mathrm{r}}$ (see Eq.(3)) and thus modify at will the phase of the Ramsey fringe pattern described by equation (4).

In next section, we will analyse in simple terms optimal procedures aiming at determining $n$ as fast as possible by taking advantage of the possibility of varying at will $\varphi$ and $\varepsilon$ during each measurement sequence. We will start by discussing simple situations where the photon number is very small. 


\section{Optimal measurement of small photon numbers.}

We will assume first for simplicity that the photon number in the cavity does no exceed a few units. Practically this can be achieved by coupling the cavity for a finite time with an external source, either coherent or thermal, then switching off the coupling and letting the field relax freely and slowly. The cavity walls are supposed to be thermalized at a temperature so low that the average number of thermal photons in steady state is negligible. The number of photons initially present can be adjusted by varying the source intensity and the duration of its coupling to the cavity.

3.1 ZERO OR ONE PHOTON IN THE CAVITY. - Suppose first that $n$ can take only the two values 0 or 1 . For example, if we prepare in the cavity a coherent field with an average photon number $\bar{n}=0.2$ the probabilities of finding 0 and 1 photon are respectively 0.82 and 0.16 and the probability of detecting more than one photon is 0.0175 , which realizes a good approximation of a two-photon state. In order to measure such a double-valued quantity, we only need to detect one atom. If we choose $\varphi=0$ ( exactly resonant Ramsey fields) and $\varepsilon=\pi$, the probabilities of detecting the atom in $f$ or $e$ (given by Eq.(4)) take the simple values 0 and 1 , so that we can unambiguously assign for each photon number a different outcome for the atomic measurement, according to table I.

Table I. - Outcome of atom measurement for a two-photon-state field.

$\begin{array}{cc}\text { Photon Number } & \text { Detected Atomic } \\ n & \text { State } \\ 0 & \mathrm{f} \\ 1 & \mathrm{e}\end{array}$

Thus, the detection of atom in $\mathrm{f}$ means that $n=0$ and the detection of this atom in $\mathrm{e}$ projects the photon number in the $n=1$ Fock state. In the example chosen above (coherent field with $\bar{n}=0.2$ ), we will find about 4 out of 5 times the atom in state $f$ and one in five time in e. Once the first atom has been measured, all subsequent atoms are, in the case of an ideal non relaxing cavity, detected with certainty in the same state as the first one since $n$ cannot change any longer (complete correlation of atomic events in this simple case). This of course implies that the same values of the parameters $\varphi$ and $\varepsilon$ are used for all atoms. In a real cavity, each reduction of the field into the $n=1$ photon state is followed by its eventual relaxation to the vacuum state, due to unavoidable cavity damping. As soon as the photon disappear, the "atomic digit"jumps back from e to $f$. We thus expect to measure either an indefinite ffffff...ff sequence (field measured in the vacuum state and remaining in it) or a sequence of the form eeeeeffff.... ff, i.e. a finite sequence of e "digits" (signalizing the existence of just one photon in the cavity) followed by an infinite sequence of $f$ digits (the field has relaxed down to vacuum). The abrupt change from the e to value is a field quantum jump, directly observable on the atomic variables, which represents the "death of a photon" in the cavity. The exact time of this event is, in a given sequence, unpredictable. If one resumes the sequence over and over, the statistical distribution of the decay times reconstructs the usual exponential law of the cavity energy relaxation. 
3.2 ZERO, ONE OR TWO PHOTONS IN THE CAVITY. - Let us now complicate a little bit this probability game and assume that the photon number can take the values 0,1 or 2 only. We now need to detect at least two atoms in order to measure $n$. We can easily show that 2 is in fact a sufficient number. Let us choose for atom(1) $\varphi_{1}=0$ and $\varepsilon_{1}=\pi$ and for atom(2) $\varphi_{2}=0$ and $\varepsilon_{2}=\pi / 2$. The probabilities of detecting each of these atoms in levels $f$ or e are again given by equation (4) and the possible outcomes of the measurement for the two atoms are listed in table IIa ( when the two outcomes are possible, they are in fact equally probable in this case)

Table IIa. - Possible outcomes of atoms measurement for a three-photon state field.

Photon Number
$n$
0
1
2

Atom (1)
$\left(\varphi_{1}=0, \varepsilon_{1}=\pi\right)$
$\mathrm{f}$
$\mathrm{e}$
$\mathrm{f}$

Atom (2)

$\left(\varphi_{2}=0 ; \varepsilon_{2}=\pi / 2\right)$

f

e

In an experiment, we would of course have to deduce the field state from the measured "atomic digit" sequence and we must thus read the above table "backwards", listing all the possible sequence outcomes and assigning them to possible photon numbers. We immediately get, by mere inspection of table IIa, and without any ambiguity the assignements listed in table IIb below.

Table IIb. - Assignement of atomic digit sequence to photon number for three-photon statesystems.

$\begin{array}{cc}\text { Atomic Digit Sequence } & \text { Photon Number } \\ \text { (atom (1) first listed) } & n \\ \text { f, f } & \\ \text { f,e } & 0 \\ \text { e,f } & 2 \\ \text { e,e } & 1 \\ \end{array}$

There is now some unavoidable redundancy, since we have three states to recognize and four possible results of the measurement, but the important point is that we arrive in two steps to a complete measurement of the field.

3.3 FROM ZERO UP TO THREE PHOTONS IN THE CAVITY. - The last special case we will consider is the four photon state one $(n=0,1,2,3)$. Although, we will show shortly that two atoms are enough to determine $n$, this will require some trick and it is easier first to keep playing the relatively simple game we have illustrated above on the two and three state cases. Let us detect now three atoms, each corresponding to a different setting of the $[\varphi, \varepsilon]$ parameters. We choose $\varphi_{1}=0, \varepsilon_{1}=\pi \varphi_{2}=0, \varepsilon_{2}=\pi / 2$ and $\varphi_{3}=\pi / 2, \varepsilon_{3}=\pi / 2$. The table of possible outcomes for each atom, based on the probabilities given by equation (4) is (here again, when the two outcomes are possible, they are equally probable): 
Table IIIa. - Possible outcomes of atoms measurements for a four photon-state field.

$\begin{array}{cccc}\text { Photon Number } n & \text { Atom (1) } & \text { Atom (2) } & \text { Atom (3) } \\ 0 & \text { f } & \text { f } & \text { f or e } \\ 1 & \text { e } & \text { f or e } & \text { f } \\ 2 & \text { f } & \text { e } & \text { f or e } \\ 3 & \text { e } & \text { f or e } & \text { e }\end{array}$

Table IIIb. - Assignment of atomic digit sequences to photon number for four-photon-state systems.

Atomic digit sequence Photon number $n$

$\begin{array}{ll}\mathrm{f}, \mathrm{f}, \mathrm{f} & 0 \\ \mathrm{f}, \mathrm{f}, \mathrm{e} & 0 \\ \mathrm{f}, \mathrm{e}, \mathrm{f} & 2 \\ \mathrm{f}, \mathrm{e}, \mathrm{e} & 2 \\ \mathrm{e}, \mathrm{f}, \mathrm{f} & 1 \\ \mathrm{e}, \mathrm{f}, \mathrm{e} & 3 \\ \mathrm{e}, \mathrm{e}, \mathrm{f} & 1 \\ \mathrm{e}, \mathrm{e}, \mathrm{e} & 3\end{array}$

We now list the eight possible outcomes of the triple atom measurement, with the photon assignement we again arrive at after simple inspection of the above table:

Here again, the photon assignment is unambiguous but now each photon number corresponds to two distinct atomic sequences so that it is legitimate to ask whether is it possible to do better and to detect the same field with two atoms only (which yield four different outcomes). This can indeed be achieved in the following way. We still have three atoms at our disposal, each with the different set of $[\varphi, \varepsilon]$ parameters given above, but now we send and detect atom(1) first and, depending upon the outcome, decide to send either atom (2) or atom (3). If atom (1) is detected in $f$ we know from table IIIa that the field contains zero or two photons. $\operatorname{Atom}(2)$ is then obviously the right choice to try since its detection will allow us to discriminate unambiguously between $n=0$ and $n=2$. If the second atom is found in $\mathrm{f}$, the answer is necessarily $n=0$ and it is $n=2$ if it is found in e. On the other hand, an e outcome for atom(1) reduces the field to a mixture of $n=1$ and $n=3$, for which atom(3) is discriminant. If it is found in $f$, the answer is $n=1$ and it is $n=3$ if it is found in e. These results are summarized in the following table which lists all the possibilities.

3.4 GENERALIZATION TO LARGER PHOTON NUMBERS: DESCRIPTION OF AN OPTIMAL PROCEDURE. - This discussion shows that the photon number measurement can be performed according to different procedures. We have described above a "passive" method, in which the atomic sequence is chosen once for all, with preset values of the parameters $[\varphi, \varepsilon]$. Of course, values have to be chosen in a clever way, so as to gain rapidly information on the field. We have also shown that an "active" method in which the parameter $\varphi$ is chosen after the previous atom has been detected is even more efficient. The idea is of course to wait until we get some information on the system in order to "ask" the "right question" which will optimally increase our information on $n$. The "active method" described above in the four photon state field case 
Table IIIc. - Measurement of a four-photon state field with two atoms.

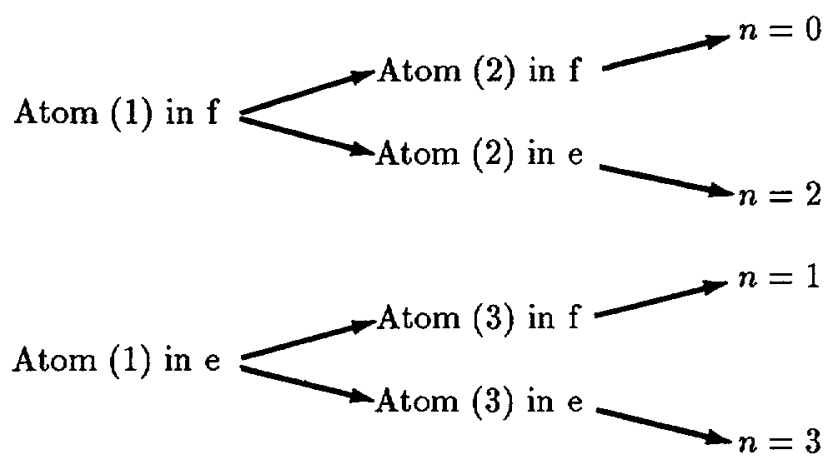

can obvioulsy be generalized to an arbitrary number of photons in the field.

The principle of the procedure is indeed simple. By choosing for the first atom $\varepsilon=\pi$, we multiply the initial (unknown) photon number distribution by a "fringe" function of $n$ having a period $\delta n=2$, which automatically decimates one out of two photon numbers. Of course, there is a degree of randomness in the process and we cannot a priori know whether we will destroy the even photon numbers (if the atom is detected in $e$ ) or the odd ones (if the atom is detected in $\mathbf{f}$ ). We then send the next atom with $\varepsilon=\pi / 2$, so as to decimate one out of four photon number states. This will be achieved, though, provided the phase of the second atom "fringe pattern" has a maximum for photon numbers which have been left after the first one, an information that we cannot have until the first atom has been detected. We thus decide, depending upon the outcome of atom (1) measurement, which phase $\varphi$ we need for the second atom. In this way, we end up with a $p(n)$ distribution in which only one out of four $n$ states survives. The third atom, if required, is then sent with $\varepsilon=\pi / 4$ and a conveniently chosen $\varphi$ value, as a result of which only one out of eight photon number survives and so on. If the width of the initial distribution is $\Delta n$, this dichotomic procedure obviously converges to a Fock state within this width after $k$ steps such that $2^{k} \geq \Delta(n)$. Two atoms are enough for $\Delta(n)=4$ as shown above. A field with an average photon number $\bar{n}=10$ has a negligible photon number probability outside the $n=0,20$ interval and will thus be measured with 5 atoms only. Clearly, it is impossible to do better than this in term of information theory. We measure indeed an integer with a binary system yielding elementary double valued answers. We thus need at least $\log _{2}(\Delta n)$ steps to perform a measurement.

We can finally compare the efficiency of the procedure we have just outlined with the one described by numerical simulations in reference [2], in which the atomic velocity was randomly varied from atom to atom. Both methods converge after a number of steps proportional to the logarithm of the number of initial photon states, but an $\bar{n}=10$ coherent state is pinned down with about 15 atoms in the random velocity case, whereas 5 atoms are enough if the optimal procedure is used. We can thus say that the random method is about three time less efficient than the ideal one. Of course, the random method may seem much simpler, since it is well adapted to an experiment using a thermal beam of atoms. We must stress however that techniques to select an atomic velocity and to tune the $\varphi$ and $\varepsilon$ parameters between consecutive atoms are not in principle difficult to implement. The gain in convergence time is not negligible in experiments where the relaxation time of the cavity may be the limiting factor. 


\section{Conclusion.}

We have shown in this article that the non resonant method of measuring the number of photons in a high $Q$ cavity which we have introduced and described in earlier references can be implemented in such a manner that the information is extracted from the field in the most efficient way possible. This requires an "intelligent" scheme in which the successive atoms are "tuned" in a way depending upon the outcomes of the detection on previous atoms, so as to "ask at each step the right question to the field". In this way, only four or five atoms are required to measure a field in which the number of photons is initially comprised between zero and twenty. An experiment using such a method could reveal in a striking way the quantum jumps of the field in the cavity, which occur suddenly at random times as the average photon number slowly relaxes to zero. We must emphasize that the method does not require that all the atoms are measured. Unread atoms, as noticed in [2] have no effect on the photon number distribution. The above arguments are valid if we consider only measured atoms and forget about those which have escaped detection. Of course other sources of noise would have to be considered in a real experiment, including false readings (an atom actually found in $\mathrm{e}$ is assigned by error to f), limited fringe contrast due to unperfect velocity selection...A more complete analysis of this problem, taking these complications into account will be presented elsewhere.

\section{References}

[1] Brune M., Haroche S., Lefevre V., Raimond J. M. and Zagury N., Phys. Rev. Lett. 65, (1990) 976.

[2] Brune M., Haroche S., Raimond J. M., Davidovich L. and Zagury N., Phys. Rev. A, to be published (1992).

[3] Haroche S., Brune M. and Raimond J. M., Optics and interferometry with Atoms, Applied Physics (Special issue, P. Meystre and J. Mlynek, editors, Springer Verlag, 1992).

[4] Carnal O. and Mlynek J., Phys. Rev. Lett 66, (1991) 2689.

[5] Ramsey N. F., Molecular Beams (Oxford University Press, New York, 1985). 